\title{
Brief Intermittent Neuroleptic Prophylaxis for Selected Schizophrenic Out-patients
}

\author{
H. A. McCLELLAND, G. HARRISON and S. D. SONI
}

\begin{abstract}
"A study was conducted to investigate a novel approach to the prophylaxis of schizophrenic relapse. The treatment strategy comprised brief intermittent courses of neuroleptic agents begun as soon as non-psychotic symptoms believed to be early signs of relapse appeared. Fifty four stable, remitted outpatients meeting the American Psychiatric Association's DSM-III criteria for schizophrenia were randomised double blind to receive brief intermittent treatment with either active or placebo depot neuroleptic injections. Only three patients given placebo injections and two controls were admitted to hospital during one year of follow up. Eight $(30 \%)$ of the patients given placebo injections and only $2(7 \%)$ of the controls, however, had a recurrence of schizophrenic symptoms. Patients given placebo injections experienced fewer extrapyramidal side effects and showed a trend towards a reduction in tardive dyskinesia. Dysphoric and neurotic symptoms were identified before eight out of 11 relapses, and these symptoms were more frequent in patients given placebo depot injections. These results suggest a viable but not necessarily better alternative to continuous oral or depot treatment for less ill, chronic, stabilised schizophrenics based on the early treatment of putative prodromal symptoms of relapse."
\end{abstract}

The summary quoted above is from an article by Jolley et al (1989). The present authors were invited to comment upon the study.

\section{H. A. McClelland}

Neuroleptic medication is an indispensable evil in the treatment of schizophrenia. Indispensable because even though there is no evidence that these drugs have increased the 'cure' rate of schizophrenia, their beneficial effects on relapse rates and control of symptoms cannot be disputed; evil because of the wide range of side-effects that can be displeasing and even a source of hardship for the patient. Understandably but unfortunately, psychiatrists have pursued the Holy Grail of achieving at all costs the maximum possible antipsychotic effectiveness of these drugs. There has been an acceptance of a loosely defined standard dosage, with frequent recourse to above-average dosage in treatmentrefractory patients, although if a steady increase in dosage does not cause the desired effect, it is fairly uncommon for the dosage to be reduced. The outcome is a range of adverse reactions to which the psychiatrist is more tolerant than the patient.

Early attempts at overall low dosage, by instituting 'drug holidays' with fixed and arbitrary intervals, all failed, but the recent upsurge of interest in continuous low-dosage or intermittent neuroleptic regimes has been carried out with more sophisticated and carefully supervised studies. It is no coincidence that systematic trials of these procedures have been performed initially in the USA. There, the increasing awareness of tardive dyskinesia and of the misery caused by side-effects in general has been forcibly drawn to the attention of psychiatrists by the vociferous advocates of patients' civil rights, accompanied by costly legal actions.

The trial by Dr Jolley and his colleagues is admirable in concept and design. That relapse is frequently preceded by dysphoric symptoms is confirmed. The overall reduction in side-effects is clearly shown, and this must increase the patient's co-operation. Some years ago, Van Putten (1974) showed that many patients were not so much non-compliant as partially compliant, in that they self-medicated at a dose level which made their side-effects bearable. Yet if a doctor does not know how much medication a patient is taking, treatment becomes a blind-man's buff.

The authors do not claim that all patients are suitable for their regime, and indeed accepted only $40 \%$ of the patients referred. They delineate certain obvious criteria for inclusion, such as lack of dangerousness in relapse and reasonable degree of pre-trial stability. This drug regime is therefore not suitable for the majority of patients with chronic schizophrenia. This agrees with a recent study (Chiles et al, 1989) on eligibility of patients for intermittent medication, where the authors found that only onethird $(34.8 \%)$ of chronic schizophrenic patients attending a mental health centre were suitable. 
In all drug trials, there is a risk that a small but distinct subgroup can be overlooked. For some patients, a relapse can have serious consequences for livelihood and domestic stability. There is also a small proportion who may have a number of relapses followed by excellent remission, but then a further relapse leads to a serious defect state. Ciompi (1980) estimates that such an outcome occurs in about $10 \%$ of patients. One cannot predict which patients belong to the subgroup, and although the relapsed patients in the Jolley trial did well with early intervention, this might not be true for all relapses over a longer period of time.

The duration of follow-up is critical in the evaluation of any management regime. Some early trials of low-dose medication were for less than a year, and even a trial of one year may not be sufficient. The relapse rates in ultra-low and standard dosage in the study by Marder et al (1987) were similar over one year but over two years, the rate on ultra-low dose was very much higher, although if the psychiatrist could alter dosages, the relapse rates were similar.

In the Jolley trial, the results over a year were acceptable, even if those on intermittent dosage did show more dysphoric symptoms and more relapses. However, if the study had been continued for a further year, deterioration of those on intermittent medication may have been unacceptable in terms of frequency and severity of relapse. The authors have properly argued that a similar trial on intermittent medication by Carpenter et al (1987) did not show any appreciable difference in the two groups over two years. Another recent trial (Hogarty et al, 1988) of continuous low dose (but not ultra-low dose) also did not show any differences in relapse rates, compared with a standard dose.

However, there is an important maverick study on continuous low dosage from Manchester (Johnson et al, 1987). Not only did those investigators find a significantly increased relapse rate at 12 months, comparing standard with reduced doses $(50 \%)$ of flupenthixol decanoate, the relapse rate increasing to $56-75 \%$ over two to three years in the reduceddose group, but the latter experienced neither improvement in social functioning nor reduction in side-effects (apart from tardive dyskinesia). The patients in this trial were stabilised out-patients, but they were entered consecutively, and could well have been less symptom-controlled than those in the Jolley trial. Assessments were carried out at three-monthly intervals, and this trial reflects more accurately the conditions of day-to-day clinical practice.

On balance, it would seem that with judicious selection of patients, the intermittent medication and other low-dose (but not ultra-low) techniques can be a significant advance in the treatment of a minority of patients with schizophrenia. Jolley et al, like most other investigators, have emphasised the fewer sideeffects, with the patient having a better quality of life overall. Such results have been obtained by enthusiastic and experienced workers after lengthy assessment (and presumably supportive) interviews with the patients at monthly intervals, and there were educational packages for patients and relatives. Chronic patients with functional psychosis (whether in good or incomplete remission) require such meticulous and frequent supervision so that modern drug, social, and counselling techniques are properly utilised. This will include the development of case registers and continuity of contact with interested therapists. It is clear that these ideal conditions do not exist in real life, except in a few centres with special resources, and they are very difficult to maintain over the long periods necessary in the management of schizophrenics.

For some years, I have endeavoured to reduce medication in my patients and am in no doubt that many do very well on smaller amounts than what are usually regarded as 'standard' doses. However, until I have enough resources to organise a foolproof community service, my preference remains for constant low dosage in carefully selected and monitored patients, rather than "intermittent neuroleptic prophylaxis", which requires a very efficient service indeed. Whatever one's choice, though, the present stereotyped drug regimes administered to all patients cannot be the best clinical practice.

\section{G. Harrison}

Throughout the history of medicine, from bloodletting to aggressive cytotoxic therapy, there have been examples of cures which may be worse than the illness being treated. While the side-effects associated with neuroleptic treatment fall far short of equivalence with the distressing personal and social sequelae of unremitting psychosis, the authors remind us of the price sometimes paid for effective prophylaxis. Graphic imagery of the "greasy masked facies, stooped posture, 'dancing feet' and slow, shuffling gait" which "mark some patients out in the crowd" readily engages our interest in any treatment approach promising effectiveness while reducing the cost to the patient.

In parallel with the search for new groups of drugs having intrinsically fewer side-effects, there have been a number of alternative strategies suggested for the delivery of those already in use. These have 
included low-dose regimes, 'drug holidays' and, most recently, brief intermittent therapy.

This is an important and carefully executed trial of a variation of brief intermittent treatment. The strategy proposed in this paper relies upon identifying a "personal style of relapse" in individual patients, characterised by the emergence of non-specific neurotic or dysphoric symptoms before relapse. Oral haloperidol is then rapidly introduced in order to prevent psychotic symptoms becoming fully established.

The authors are to be commended on their detailed and careful presentation of their findings. They candidly describe this as a study of "selected schizophrenic patients". As in many intervention studies, the subjects bore little resemblance to the majority of patients currently maintained in our clinics. In effect, they were patients who had complied with depot neuroleptics for six months before entry (and who therefore had residual blood levels during the trial), who had been stable and free of symptoms over this period, and in whom relapse had not previously entailed definite risk to self or others. Of those passing the provisional filter of referral by clinicians who thought that they might benefit from the treatment, only $40 \%$ were entered for the study. The intervention group had a higher age of onset of illness, suggesting a better prognosis overall.

At one-year follow-up, $76 \%$ of the study group had had prodromal symptoms (non-specific neurotic and dysphoric complaints), compared with only $27 \%$ in the continuous depot control group. Thirty per cent relapsed, of whom half were apparently withdrawn from blind conditions because they were either ill for more than eight weeks or had experienced two or more relapses in six months. In contrast, only $7 \%$ of the control group relapsed, although there was little difference in the need for hospital admission or in use of compulsory powers. Advantages to patients in the study group included fewer extrapyramidal side-effects, as well as a trend towards a reduction in tardive dyskinesia.

Despite the lower incidence of side-effects, the actual benefit to patients in the experimental group is difficult to quantify. Prodromal symptoms involved real suffering to patients and, following the educational package about their illness, they knew that these carried the threat of impending psychosis. While those in the study who relapsed did not usually require hospital admission, the repercussions in less compliant, less carefully selected, and less closely supervised patients in our clinics require little imagination.
To show a true advantage, a treatment package involving significantly more risk of relapse and a higher prevalence of non-psychotic symptoms must demonstrate substantially lower side-effects and longer-term advantages of improved social outcome. A week may be a long time in politics, but one year is a notoriously limited period for assessing the course and outcome of schizophrenic syndromes. The results in the second year of follow-up will demonstrate the true worth, or otherwise, of this treatment. By that time, residual depot medication will have completely cleared from patients in the intervention group, and the additional social support they required over the first year may undergo subtle changes in both its intensity and the novelty of its impact. We await follow-up data from this research team with interest. Until then, even my most 'selected patients' will remain on continuous medication.

\section{S. D. Soni}

The paper from Charing Cross Hospital, London, describes a 'novel' approach to neuroleptic prophylaxis in schizophrenia. The study has been carried out with rigorous attention to methodological detail, as reflected in its design, and has produced interesting results. Briefly, these suggest that in certain 'selected' patients, continuous maintenance neuroleptic therapy may not be necessary; instead, they could be carefully monitored following recovery from acute psychosis and at the first signs of imminent relapse, oral neuroleptics instituted for brief periods to control symptoms and abort a florid relapse. The study raises important issues both with regard to the assumptions on which the study is based and inferences drawn from the findings.

The idea of brief intermittent neuroleptic prophylaxis ('targeted pharmacotherapy') for relapse in schizophrenia was introduced because of concern about the adverse effects of long-term medication, especially the risk of tardive dyskinesia (Herz et al, 1982; Carpenter \& Heinrichs, 1983). The approach involved establishing criteria for selection of patients, tapering of medication, careful monitoring for prodromal signs of exacerbation, and restarting medication if indicated by early signs of relapse (Chiles et al, 1989). The assumption behind this strategy is that in many schizophrenics, the relapse occurs in stages (Docherty et al, 1978; Herz \& Melville, 1980) so that it might be aborted before the development of a florid psychotic state. Previous studies have confirmed that this is possible, but that it requires careful selection of patients (Herz et al, 1982). The patients selected for this study were stable 
schizophrenics on maintenance depot neuroleptics, but few other criteria are mentioned, except that the treating clinicians thought they would be suitable for the study. Chiles et al (1989) have in fact indicated specific exclusion criteria for their 'intermittent medication' regime, such as presence of baseline symptoms, current stressful life events, uncooperativeness, hospital admission in the previous three months, suicidal behaviour, use of other psychotropic medication, etc. Further research should crystallise these observations and establish firm criteria, so that the findings could be translated into clinical practice.

Pharmacokinetic studies on depot neuroleptics reveal a prolonged and variable elimination rate, especially for fluphenazine decanoate; this can be an important confounding variable which, quite appropriately, has been discussed by the authors but rejected as clinically insignificant. The abrupt withdrawal of fluphenazine decanoate in this report also differs from the design of other similar studies, which recommend gradual tapering of the therapy. Finally, patients included in this study were stable schizophrenics; it is, however, well established that follow-up of treated schizophrenics in the first $\mathbf{1 2}$ months after recovery from acute psychotic episode reveals much higher rates of relapse than follow-up of similar patients who have been stable for a year or more on maintenance neuroleptic treatment (Hogarty, 1984). The findings in the present study therefore may not be wholly applicable to patients who have just recovered from a clinical episode of acute psychosis.

An important factor in assessing the value of this study concerns the nature of relapse. A key assumption in it is that the non-specific symptoms which were seen in patients were in fact early signs of schizophrenic relapse, but this is by no means established. As the authors quite rightly indicate, schizophrenics often show dysphoric symptoms in response to adverse life events, which may or may not predict an imminent relapse. Many schizophrenics also continue to show intermittent or sustained affective and/or neurotic symptoms while on stable doses of neuroleptics, and it is imperative that the relationship of these symptoms to relapses is carefully investigated.

An aspect of clinical significance is both the immediate and long-term effects of relapses on the handicaps of schizophrenic illness. One reason for maintaining patients on neuroleptic therapy is to prevent distress to both the patient and his relatives which is caused by excacerbation of acute psychosis. This aspect may certainly be reduced, but never eliminated, in some patients with the use of brief intermittent therapy. In our choice of approach for individual patients, these factors may have to be carefully balanced. Another consideration is the uncertainty about the relationship between severity and frequency of relapses and the eventual emergence and severity of deficits (including negative symptoms). If such a relationship exists, then the benefits obtained by reduced exposure to neuroleptics by the brief intermittent therapy recommended by this study may well be outweighed by the development of a more severe defect state and the consequences this may entail for the functioning of the patient. These aspects certainly need further investigation.

A final point relates to cost-effectiveness: maintenance neuroleptic therapy is relatively inexpensive; continuous, careful monitoring of patients, on the other hand, requires much staff time and vigilance, as well as active involvement and education of relatives or carers. These factors need to be carefully evaluated, especially in terms of cost/benefit analysis before the strategy proposed could be generally accepted, since selection of patients and the long-term effects on schizophrenics of repeated (albeit abortive) relapses still require further investigation. Follow-up of the present cohort may clarify some of these points, and we look forward to further reports from this centre.

\section{References}

Carpenter, W. T. \& Heinrichs, D. W. (1983) Early intervention, time-limited, targeted pharmacotherapy of schizophrenia. Schizophrenia Bulletin, 9, 533-542.

- _ \& HANLON, T. E. (1987) A comparative trial of pharmacological strategies in schizophrenia. American Journal of Psychiatry, 144, 1466-1470.

Chiles, J. A., Sterchi, D., Hyde, T., et al (1989) Intermittent medication for schizophrenic outpatients: who is eligible? Schizophrenia Bulletin, 15, 117-120.

Ciompi, L. (1980) Catamnestic long-term study on the course of life and aging of schizophrenics. Schizophrenia Bulletin, 6 , 606-618.

Docherty, J. P., Van Kammen, D. P., Siris, S. G., et al (1978) Stages of onset of schizophrenic psychosis. American Journal of Psychiatry, 135, 420-426.

Herz, I. M. \& Melville, C. (1980) Relapse in schizophrenia. American Journal of Psychiatry, 137, 801-805.

-, SzYmanski, H. V. \& Simon, J. C. (1982) Intermittent medication for stable schizophrenic outpatients: an alternative to maintenance medication. American Journal of Psychiatry, 139, 918-922.

HogarTY, G. E. (1984) Aftercare treatment of schizophrenia. In Seminar on Schizophrenia (ed. S. R. Hirsch), pp. 38-42. London: Update.

- McEvoy, J. P., Munetz, M., et al (1988) Dose of fluphenazine, familial expressed emotion, and outcome in schizophrenia. Archives of General Psychiatry, 45, 797-805. 
Johnson, D. A. W., Ludlow, J. M., Street, K., et al (1987) Double-blind comparison of half-dose and standard-dose flupenthixol decanoate in the maintenance treatment of stabilized out-patients with schizophrenia. British Journal of Psychiatry, 151, 634-638.

Jolley, A. G., Hirsch, S. R. \& Manchanda, R. (1989) Trial of brief intermittent neuroleptic prophylaxis for selected schizophrenic out-patients: clinical outcome at one year. British Medical Journal, 293, 985-990.

Marder, S. R., Van Putten, T., Mintz, J., (1987) Low- and conventional-dose maintenance therapy with fluphenazine decanoate. Archives of General Psychiatry, 44, 518-521.

VAN Putren, T. (1974) Why do schizophrenic patients refuse to take their drugs? Archives of General Psychiatry, 31, 67-72.

H. A. McClelland, MB, BS, FRCP, FRCPsych, Consultant Psychiatrist, St Nicholas Hospital, Gosforth, Newcastle-upon-Tyne NE3 3XT; Glynn Harrison, MB, ChB, MRCPsych, Consultant Psychiatrist, Academic Department of Psychiatry, University Hospital, Nottingham NG7 2UH; Som D. Soni, MD, PhD, FRCP, FRCPsych, Consultant Psychiatrist, Salford Health Authority, Hope Hospital, Eccles Old Road, Salford M6 8HD 